\title{
EQUIVALENCE IN A CLASS OF DIVISION ALGEBRAS OF ORDER 16
}

\author{
R. D. SCHAFER
}

Let (5) be a Cayley-Dickson division algebra over an arbitrary field $\mathfrak{F}$ with principal equation

$$
x^{2}-t(x) x+n(x)=0
$$

and involution

$$
S: \quad x \leftrightarrow x S=t(x)-x .
$$

We are concerned with division algebras $\mathfrak{A}$ of order 16 over $\mathfrak{F}$ defined in the following way: let $\mathfrak{G}_{0}$ be a division algebra (of order 8 ) over $\mathfrak{F}$ with the same elements as $\mathbb{E}$ but with multiplication denoted by xoy; further let $\mathfrak{A}=\mathfrak{E}+v \mathfrak{E}$, multiplication ${ }^{1}$ in $\mathfrak{A}$ being defined by

$$
c z=(a+v b)(x+v y)=(a x+y o b S)+v(a S \cdot y+x b)
$$

for $a, b, x y$, in $\mathfrak{C}$.

In the original form of this paper, the author considered the problem of equivalence in the class of algebras $\mathfrak{A}=\mathfrak{C}+v \mathfrak{E}$ with multiplication defined by

$$
c z=(a+v b)(x+v y)=(a x+g \cdot y b S)+v(a S \cdot y+x b)
$$

for $a, b, x, y$ in $\mathbb{E}$ where $g$ is a fixed element of $\mathfrak{E}, g \in \mathfrak{F}$. The author had shown in [5] that $\mathfrak{A}$ is a division algebra in case $g$ is chosen with $n(g)$ not a square in $\mathfrak{F}$; in particular, such a choice of $g$ can be made when $\mathfrak{F}$ is the field $R$ of rational numbers. R. H. Bruck, the referee of the paper in its original form, suggested a study of the wider class of algebras defined by (3). Theorems 1 and 2 are generalizations of the result in [5] and are due ${ }^{2}$ to R. H. Bruck. By their use the class of algebras studied here has been considerably enlarged. ${ }^{3}$

In $\$ 2$ we shall determine conditions for the equivalence of two alge-

Presented to the Society, April 27, 1946; received by the editors February 26, 1946, and, in revised form, May 29, 1946.

1 This modification of the Cayley-Dickson process was originally presented by R. H. Bruck in [2], Theorem $16 \mathrm{C}$, to obtain non-alternative division algebras of orders 4 and 8 . Numbers in brackers refer to the references cited at the end of the paper.

2 Theorem 2 was communicated to the author by Bruck, complete except for the proof of the equivalence of $\mathfrak{E}_{*}$ and $\mathfrak{E}$, a fact which Bruck conjectured.

${ }^{3}$ See the comment following the corollary to Theorem 4. 
bras $\mathfrak{A}, \mathfrak{A}_{*}$ of this class. These conditions lead directly to a determination of the automorphisms of $\mathfrak{A}$.

1. A class of division algebras of order 16 . An algebra $\mathfrak{Q}$ is called a quaternion algebra if $\mathfrak{Q}=\left(1, u_{2}, u_{3}, u_{4}\right), u_{4}=u_{3} u_{2}, u_{2}{ }^{2}=u_{2}+\alpha, u_{3}{ }^{2}=\beta$, $u_{2} u_{3}=u_{3}\left(1-u_{2}\right)$, where $\alpha$ and $\beta \neq 0$ are in $\mathfrak{F},-4 \alpha \neq 1$. An algebra $\mathbb{E}$ is called a Cayley-Dickson algebra if $\mathfrak{S}=\mathfrak{Q}+u_{5} \mathfrak{Q}$, with elements $x=p+u_{5} q$, where $p, q$ are quaternions and multiplication is defined by $\left(p_{1}+u_{5} q_{1}\right)\left(p_{2}+u_{5} q_{2}\right)=\left(p_{1} p_{2}+\gamma q_{2} \cdot q_{1} S\right)+u_{5}\left(p_{1} S \cdot q_{2}+p_{2} q_{1}\right)$ where $u_{5}{ }^{2}=\gamma$ $\neq 0$ in $\mathfrak{F}$ and $S$ is the involution (2) of $\mathfrak{Q}$.

Among the well known properties of $\mathfrak{S}$ which we shall use are that $(\mathbb{S}$ is an alternative algebra (see [4]), that $\mathbb{E}$ is central simple, that (1) holds for $x$ in $\mathfrak{E}$, where

$$
x+x S=t(x), \quad x(x S)=(x S) x=n(x)
$$

for the involution (2) of $\mathfrak{c}$. The norm form $n(x)$ permits composition-that is, $n(x y)=n(x) n(y)$ for $x, y$ in (E.

Theorem 1. Let (5) be a Cayley-Dickson division algebra over $\mathfrak{F}$ with involution $S$. Let $\mathfrak{A}=\mathfrak{C}+v \mathfrak{C}$ have multiplication defined by (3), where $\mathfrak{G}_{0}$ is chosen so that

$$
n(x \circ y)=\lambda n(x) n(y), \quad \lambda \neq 0 \text { in } \mathfrak{F},
$$

for all $x, y$ in $\mathfrak{C}$. If $\lambda$ is not a square in $\mathfrak{F}$, then $\mathfrak{A}$ is a division algebra over $\mathfrak{F}$.

A necessary and sufficient condition that $\mathfrak{A}$ be a division algebra is that

$$
a x+y o b S=0, \quad a S \cdot y+x b=0 \quad(a, b, x, y \text { in (5) }
$$

imply either $c=0$ or $z=0$. If we assume on the contrary that ( 7 ) hold for nonzero $c, z$ it follows that $a, b, x, y$ are all nonzero. Use of (5) then shows (7) essentially equivalent to $y o b S=a[(a S \cdot y) b S] / n(b)$. Taking norms, we get

$$
n(y \circ b S)=[n(a) / n(b)]^{2} n(y) n(b S) .
$$

If $\lambda$ in (6) is not a square in $\mathfrak{F}$, then (8) cannot be satisfied for any $a, b, y$ in $\mathfrak{E}$, and $\mathfrak{A}$ is a division algebra over $\mathfrak{F}$.

In order to complete the proof of Theorem 2 we require two simple lemmas concerning Cayley-Dickson algebras.

Lemma 1. Let $\mathfrak{5}$ and $\mathfrak{F}_{*}$ be Cayley-Dickson algebras with respective 
unity elements 1 and e and principal equations $x^{2}-t(x) x+n(x) 1=0$ and

$$
x * x-t_{*}(x) x+n_{*}(x) e=0 .
$$

If $\mathbb{S}$ and $\mathfrak{E}_{*}$ are equivalent, the equivalence being given by $x \leftrightarrow x P, x$ in $\mathfrak{S}_{*}$, $x P$ in $\mathbb{E}$, then

$$
S_{*} P=P S
$$

where $S_{*}$ is the involution

$$
S_{*}: \quad x \leftrightarrow x S_{*}=t_{*}(x) e-x
$$

of $\mathfrak{E}_{*}$ and $S$ is the involution (2) of $\mathfrak{S}$.

For (9) implies that $(x * x) P-t_{*}(x) x P+n_{*}(x) e P=(x P)^{2}-t_{*}(x) x P$ $+n_{*}(x) 1=0$ since $e P=1$. But $(x P)^{2}-t(x P) x P+n(x P) 1=0$. Hence

$$
t_{*}(x)=t(x P), \quad n_{*}(x)=n(x P) .
$$

But then $\left(x S_{*}\right) P=\left[t_{*}(x) e-x\right] P=t_{*}(x) 1-x P=t(x P)-x P=(x P) S$ for all $x$ in $\mathfrak{S}_{*}$, or $S_{*} P=P S$.

LemMa 2. Let $\mathfrak{S}$ and $\mathfrak{G}_{*}$ be Cayley-Dickson division algebras over $\mathfrak{F}$ with norm functions $n(x)$ and $n_{*}(x)$. If

$$
n(x * y)=n(x) n(y), \quad x, y \text { in } \mathfrak{c},
$$

then

$$
n_{*}(x)=n(x) .
$$

We resort to the matrix representation of quadratic forms, regarding $x$ as a one-rowed matrix, so that the matrix product $x A x^{\prime}=n(x)$, where $A$ is an $8 \times 8$ matrix with elements in $\mathfrak{F}$ (' denoting transpose). Let $R_{y}, R_{y}{ }^{*}$ be the right multiplications defined by $x R_{y}=x y$, $x R_{y}^{*}=x * y$. Then $n(x * y)=n(x y)$ or $x R_{y}^{*} A R_{y}{ }^{* \prime} x^{\prime}=x R_{y} A R_{y}^{\prime} x^{\prime}$, from which it follows that $R_{y}^{*} A R_{y}^{* \prime}-R_{y} A R_{y}^{\prime}$ is a skew-symmetric matrix, and $R_{y}^{*}\left(A+A^{\prime}\right) R_{y}^{* \prime}=R_{y}\left(A+A^{\prime}\right) R_{y}^{\prime}$. Taking determinants, and noting that $\left|A+A^{\prime}\right| \neq 0$ since $\mathfrak{S}$ is a division algebra, we obtain $\left|R_{y}^{*}\right|^{2}=\left|R_{y}\right|^{2}$ or $\left[n_{*}(y)\right]^{8}=[n(y)]^{8}$. Thus $n_{*}(y)=\epsilon n(y), \epsilon^{8}=1$. Take $y=1$. Then $\epsilon=1$ and (14) follows.

THEOREM 2. Let $\mathfrak{S}$ be a Cayley-Dickson division algebra over $\mathfrak{F}$ with unity element 1 , and $\mathfrak{S}_{o}$ be a division algebra with the same elements as $\left(\mathfrak{S}\right.$ and with multiplication xoy. Then (6) holds if and only if, first, $\mathfrak{S}_{o}$ is isotopic to $\mathbb{S}$ (that is, there exist nonsingular linear transformations $U, V, W$ on (S such that

$$
x o y=(x U \cdot y V) W
$$


for $x, y$ in (E) and, second, 4

$$
n(x T)=n(x) n(1 T)
$$$$
T=U, V, W \text {. }
$$

If $\mathfrak{A}=\mathfrak{C}+v \mathfrak{S}$, multiplication defined by (3), (15), (16), and if $n(1 U) n(1 V) n(1 W)$ is not a square in $\mathfrak{F}$, then $\mathfrak{A}$ is a division algebra over $\mathfrak{F}$.

Define two nonsingular linear transformations $H, J$ on $\sqrt{ }$ in the following way: let $x H=x o 1, x J=1 o x$, for $x$ in 5 . Then, if (6) holds, $n\left(x H^{-1} o 1\right)=n(x)=\lambda n\left(x H^{-1}\right)$ and

$$
n\left(x H^{-1}\right)=n(x) / \lambda .
$$

Also $n\left(1 \circ x H J^{-1}\right)=n(x H)=n(x \circ 1)=\lambda n(x)=\lambda n\left(x H J^{-1}\right)$ or

$$
n\left(x \mathrm{HJ}^{-1}\right)=n(x) \text {. }
$$

Let $\mathfrak{G}_{*}$ be the isotope of $\mathfrak{S}_{o}$ defined by

$$
x * y=\left[x \circ\left(y H J^{-1}\right)\right] H^{-1} .
$$

Then 1 is a unity element for $\mathfrak{S}_{*}$. Moreover, $n(x * y)=n\left[x o\left(y H J^{-1}\right)\right] / \lambda$ $=n(x) n\left(y H J^{-1}\right)=n(x) n(y)$, or (13) holds. That is, $n(x)$ is a quadratic form permitting composition with respect to the multiplication $x * y$ of $\mathfrak{F}_{*}$; hence $\mathfrak{E}_{*} \quad \mathfrak{F}_{*}$ is a Cayley-Dickson algebra over $\mathfrak{F}$ and $n(x)$ is equivalent in $\mathfrak{F}$ to the norm form $n_{*}(x)$ of $\mathfrak{E}_{*}$. (Actually $n(x)$ is identical with $n_{*}(x)$ by (14), but that fact is immaterial at this point of the proof.) Since any two Cayley-Dickson algebras with equivalent norm forms are equivalent, ${ }^{6}$ it follows that $\mathfrak{C}_{*} \cong \mathfrak{C}$ with

$$
x * y=(x U \cdot y U) U^{-1},
$$

for $U$ a nonsingular linear transformation on $\mathfrak{E}$. Moreover, $\mathbb{E}_{0}$ is isotopic to $\mathfrak{S}$. Let

$$
V=J H^{-1} U, \quad W=U^{-1} H .
$$

Then (15) follows from (19), (20), (21). Also (16) holds, for $n(x U)$ $=n_{*}(x)=n(x)$ by (12) and (14), while $1 U=1=n(1 U)$; also $n(x V)$

${ }^{4}$ Any nonsingular linear transformation $T$ satisfying (16) is closely related to a norm-preserving transformation $U$. For set $1 T=t$ and $U=T R_{t}^{-1}$. Then $T=U R_{t}$ and $x T=x U \cdot t, n(x T)=n(x U) n(t)$. Hence $n(x U)=n(x)$. Conversely if $T=U R_{t}$ where $U$ is norm-preserving, then $T$ has property (16).

${ }^{5}$ See the principal theorem of $[1$, p. 161], for the relationship between quadratic forms permitting composition and the norm forms of certain alternative algebras.

${ }^{6}[3$, p. 777]. The proof in Jacobson's paper is for $\mathfrak{F}$ of characteristic not two. The author has verified the theorem for arbitrary $\mathfrak{F}$, but will not take the space to include a proof here. 
$=n\left(x J H^{-1} U\right)=n\left(x J H^{-1}\right)=n(x)$ by (18) while $1 V=1=n(1 \mathrm{~V})$, and $n(x W)=n\left(x U^{-1} H\right)=\lambda n\left(x U^{-1}\right)=\lambda n(x)$ by (17) while $n(1 W)=\lambda n(1)$ $=\lambda$.

Conversely, if multiplication in $\mathfrak{G}_{o}$ is defined by (15) and (16), then (6) holds. For $n(x o y)=n[(x U \cdot y V) W]=n(x U \cdot y V) n(1 W)$ $=n(x U) n(y V) n(1 W)=\lambda n(x) n(y)$ where $\lambda=n(1 U) n(1 V) n(1 W)$. By Theorem 1 , a sufficient condition that $\mathfrak{A}$ be a division algebra is that $\lambda$ be not a square in $\mathfrak{F}$.

2. Equivalence in this class of algebras. The algebras of $\$ 1$ are quite general, and are at the same time a concrete realization of the hypotheses in the theorems of this section. In order to show that these theorems, which are actually proved for a more general class of division algebras, apply to the algebras described in $\S 1$, we note that if $\mathfrak{E}$ is a Cayley-Dickson division algebra over $\mathfrak{F}$, and $\mathfrak{A}=\mathfrak{C}+v \mathfrak{E}$ has multiplication defined by (3) and (6), $\lambda$ not a square in $\mathfrak{F}$, then, for any nonzero element $y$ of $\mathfrak{E}$, the product yoy $S$ is not in $\mathfrak{F}$. For if yoy $S \in F$, then $(y o y S)^{2}=n($ yoy $S)=\lambda n(y) n(y S)=\lambda[n(y)]^{2}$, and $\lambda$ is a square in $\mathfrak{F}$, a contradiction.

THEOREM 3. Let $\mathfrak{A}=\mathfrak{C}+v \mathfrak{C}$, with multiplication defined by (3) where yoyS is not in $\mathfrak{F}$ for nonzero $y$ in $\mathfrak{E}$. Then an element $z$ of $\mathfrak{A}$ satisfies a quadratic equation with coefficients in $\mathfrak{F}$ if and only if $z$ is in $(\mathfrak{S}$.

For $z^{2}=(x+v y)^{2}=\left(x^{2}+y o y S\right)+v(x S \cdot y+x y)=t(x) x-n(x)+y o y S$ $+t(x) v y=t(x) z-n(x)+y o y S$. If $z^{2}-T(z) z+N(z)=0$ for some $T(z)$ and $N(z)$ in $\mathfrak{F}$, then $t(x) z-n(x)+y o y S=T(z) z-N(z)$, or $\{t(x)$ $-T(z)\} z=n(x)-y o y S-N(z)$ in $\mathbb{E}$. Either $z$ is in $\mathbb{E}$, or $t(x)-T(z)$ $=0=n(x)-y o y S-N(z)$ and $y o y S=n(x)-N(z)$ in $\mathfrak{F}$, contrary to hypothesis. Therefore the only elements of $\mathfrak{A}$ satisfying quadratic equations are the elements $z=x$ of $\mathbb{E}$.

Let $\mathfrak{A}_{*}=\mathfrak{C}_{*}+v^{\prime} * \mathfrak{C}_{*}$ where $\mathfrak{C}_{*}$ is a Cayley-Dickson algebra with principal equation (9), $\mathfrak{S}_{1}$ is a division algebra with the same elements as $\mathfrak{E}_{*}$ but with multiplication $[x, y]$. Let multiplication in $\mathfrak{A}_{*}$ be defined by

$$
\left(a+v^{\prime} * b\right) *\left(x+v^{\prime} * y\right)=\left\{a * x+\left[y, b S_{*}\right]\right\}+v^{\prime} *\left(a S_{*} * y+x * b\right)
$$

for $a, b, x, y$ in $\mathfrak{E}_{*}$, where $S_{*}$ is the involution (11).

THEOREM 4. A division algebra $\mathfrak{A}_{*}=\mathfrak{C}_{*}+v^{\prime} * \mathfrak{C}_{*}$ with multiplication defined by (22), $\left[y, y S_{*}\right]$ not in $\mathfrak{F}$ for nonzero $y$ in $\mathfrak{E}_{*}$, is equivalent to a division algebra $\mathfrak{A}=\mathfrak{C}+v \mathfrak{C}$ with multiplication defined by (3), yoyS not in $\mathfrak{F}$ for nonzero $y$ in $\mathfrak{\mathfrak { S }}$, if and only if, first, $\mathfrak{E}_{*} \cong \mathfrak{E}$, the equivalence being given by $x \leftrightarrow x P, x$ in $\mathfrak{C}_{*}, x P$ in $\mathfrak{夭}$, and, second, $\mathfrak{C}_{1} \cong \mathfrak{C}_{0}$ by the 
specific equivalence $x \leftrightarrow \delta^{2} x P, x$ in $\mathfrak{G}_{1}, \delta^{2} x P$ in $\mathfrak{E}_{o}$, for some $\delta \neq 0$ in $\mathfrak{F}$. (This second condition may be stated as

$$
[x, y] P=\delta^{2}(x P o y P)
$$

for some $\delta \neq 0$ in $\mathfrak{F}$.) The equivalence between $\mathfrak{A}_{*}$ and $\mathfrak{A}$ is $x+v^{\prime} * y$ $\leftrightarrow x P+\delta v(y P)$.

Suppose $\mathfrak{A}_{*} \cong \mathfrak{A}$. Since $\mathfrak{A}_{*}$ contains a Cayley-Dickson subalgebra $\mathfrak{G}_{*}$ containing all of the elements of $\mathfrak{A}_{*}$ which satisfy quadratic equations with coefficients in $\mathfrak{F}$, it follows that $\mathfrak{E}_{*} \cong \mathfrak{E}$. Let the equivalence $H$ between $\mathfrak{A}_{*}$ and $\mathfrak{A}, z \leftrightarrow z H, z$ in $\mathfrak{P}_{*}, z H$ in $\mathfrak{A}$, have matrix

$$
H=\left(\begin{array}{ll}
P & Q \\
T & D
\end{array}\right)
$$

Then $Q=0$. For if $a \in \mathbb{E}_{*}$, both $a$ and $a H$ satisfy quadratic equations with coefficients in $\mathfrak{F}, a H \in \mathbb{S}$ and $Q=0$ in (24). The nonsingularity of $H$ implies that $P$ and $D$ are nonsingular. Moreover, the equivalence $H$ between $\mathfrak{A}_{*}$ and $\mathfrak{A}$ induces the equivalence $P$ between $\mathfrak{E}_{*}$ and $\mathfrak{S}$.

Let $L_{x}, L_{x}^{*}, R_{y}, R_{y}^{*}$ be the left and right multiplications of $\mathbb{S}$ and $\mathfrak{S}_{*}$ defined by $x y=y L_{x}=x R_{y}, x * y=y L_{x}{ }^{*}=x R_{y}^{*}$. Then multiplication is defined in $\mathfrak{A}$ by $c z=c R_{z}$,

$$
R_{z}=\left(\begin{array}{ll}
R_{x} & S R_{y} \\
S L_{y}^{(o)} & L_{x}
\end{array}\right), \quad a L_{y}^{(o)}=y o a,
$$

and in $\mathfrak{A}_{*}$ by $c * z=c R_{z}^{*}$,

$$
R_{z}^{*}=\left(\begin{array}{ll}
R_{x}^{*} & S_{*} R_{y}^{*} \\
S_{*} L_{y}^{(1)} & L_{x}^{*}
\end{array}\right), \quad a L_{y}^{(1)}=[y, a],
$$

where $R_{x}^{*}=P R_{x P} P^{-1}, L_{x}^{*}=P L_{x P} P^{-1}$, and so on.

The equivalence of $\mathfrak{A}_{*}$ and $\mathfrak{A}$ is given by $R_{z}^{*} H=H R_{z H}$ for all $z$ in $\mathfrak{A}_{*}$, or

$$
\left(\begin{array}{ll}
P R_{x P} P^{-1} & S_{*} P R_{y P} P^{-1} \\
S_{*} L_{y}^{(1)} & P L_{x P} P^{-1}
\end{array}\right)\left(\begin{array}{ll}
P & 0 \\
T & D
\end{array}\right)=\left(\begin{array}{ll}
P & 0 \\
T & D
\end{array}\right)\left(\begin{array}{ll}
R_{x P+y T} & S R_{y D} \\
S L_{y D}^{(o)} & L_{x P+y T}
\end{array}\right)
$$

for all $x, y$ in $\mathfrak{E}_{*}$.

It follows from (25) that $P R_{x P}+S_{*} P R_{y P} P^{-1} T=P R_{x P+y T}$ or $S_{*} P R_{y P} P^{-1} T=P R_{y T}$. Then (10) implies that $P S R_{y P} P^{-1} T=P R_{y T}$ or $S R_{y P} P^{-1} T=R_{y T}$. Let $y=e$ and denote $e T$ by $t$. Since $e P=1$, it follows that $S P^{-1} T=R_{\theta T}=R_{t}$, and $T=P S R_{t}$. Therefore $S R_{y P} P^{-1} P S R_{t}$ $=R_{y P S R_{t}}$ or $L_{y P S} R_{t}=R_{y P S R_{t}}$. By a lemma of Moufang [4, Lemma 1], 
$L_{y P S} R_{y P S} R_{t}=R_{y P S R_{t}} L_{y P S}$. Hence $L_{y P S} R_{y P S} R_{t}=L_{y P S} R_{t} L_{y P S}$, and for all $y \neq 0, R_{y P S} R_{t}=R_{t} L_{y P S}$.

If $t \neq 0$, let $y=t P^{-1}$. Then $R_{t S} R_{t}=R_{t} L_{t S}=n(t) I=R_{t} R_{t S}$ and $L_{t S}=R_{t S}, t=\xi$ in $\mathfrak{F}$. That is, if $t \neq 0$, then $\xi R_{y P S}=\xi L_{y P S}, R_{y P S}=L_{y P S}$, $y P S \in \mathfrak{F}$ for every $y \neq 0$ in $\mathbb{F}_{*}$, and $P$ is singular, a contradiction. Hence $e T=t=0$ and $T=P S R_{t}=0$ in (24).

With this result and (10), we may write (25) as

$$
\left(\begin{array}{ll}
P R_{x P} P^{-1} & P S R_{y P} P^{-1} \\
P S P^{-1} L_{y}^{(1)} & P L_{x P} P^{-1}
\end{array}\right)\left(\begin{array}{ll}
P & 0 \\
0 & D
\end{array}\right)=\left(\begin{array}{ll}
P & 0 \\
0 & D
\end{array}\right)\left(\begin{array}{ll}
R_{x P} & S R_{y D} \\
S L_{y D}^{(o)} & L_{x P}
\end{array}\right)
$$

from which it follows that $P S R_{y P} P^{-1} D=P S R_{y D}$, or $R_{y P} P^{-1} D=R_{y D}$. Let $y=e$ and denote $e D$ by $d$. Since $e P=1$, it follows that $P^{-1} D=R_{e D}$ $=R_{d}$, or $D=P R_{d}$. Thus $R_{y P} P^{-1} P R_{d}=R_{y P} R_{d}$ or $R_{y P} R_{d}=R_{y P R_{d}}$. Let $y=x P^{-1}$. Then $R_{x} R_{d}=R_{x d}$ for every $x$ in $(\mathbb{E}$. Therefore $d$ is a scalar $\delta$ in $\mathfrak{F}$, or $D=\delta P, \delta \neq 0$. Thus

$$
H=\left(\begin{array}{ll}
P & 0 \\
0 & \delta P
\end{array}\right)
$$

and (26) reduces to three identities and the final relationship $P S P^{-1} L_{y}^{(1)} P=\delta^{2} P S L_{y P}^{(0)}$ or $L_{y}^{(1)} P=\delta^{2} P L_{y P}^{(0)}$ from which (23) follows.

CoROllary. Let $\mathfrak{A}=\mathbb{E}+v \mathbb{S}$ be a division algebra with multiplication defined by (4), $g \notin \mathfrak{F}$, and $\mathfrak{A}_{*}=\mathfrak{S}_{*}+\mathfrak{v}^{\prime} * \mathfrak{C}_{*}$ with multiplication defined by

$\left(a+v^{\prime} * b\right) *\left(x+v^{\prime} * y\right)=\left\{a * x+g_{1} *\left(y * b S_{*}\right)\right\}+v^{\prime} *\left(a S_{*} * y+x * b\right)$

for $a, b, x, y$ in $\mathfrak{S}_{*}$, where $S_{*}$ is the involution (11) of $\mathfrak{S}_{*}$ and $g_{1}$ is a fixed element of $\mathfrak{S}_{*}, g_{1} \in \mathfrak{F}$. Then $\mathfrak{A}_{*} \cong \mathfrak{A}$ if and only if, first, $\mathfrak{E}_{*}$ is equivalent to $\mathfrak{S}$, the equivalence being given by $x \leftrightarrow x P, x$ in $\mathbb{E}_{*}, x P$ in $\mathbb{E}$, and, second, $g_{1} P=\delta^{2} g$ for some $\delta \neq 0$ in $\mathfrak{F}$. The equivalence between $\mathfrak{A}_{*}$ and $\mathfrak{A}$ is $x+v^{\prime} * y \leftrightarrow x P+\delta v(y P)$.

For $[x, y]=g_{1} *(x * y)$ and $x o y=g(x y)$ in Theorem 4 . Then (23) becomes $\left\{g_{1} *(x * y)\right\} P=g_{1} P(x P \cdot y P)=\delta^{2} g(x P \cdot y P)$, or simply $g_{1} P=\delta^{2} g$.

By Theorem 4 we see that the class of algebras described in $\$ 1$ is actually considerably larger than that defined by (4), which was the class originally studied. For any such algebra is the particular case $U=I, V=I, W=L_{0}$ of (3), (15), (16). Even such a simple variation $\mathfrak{A}_{*}$ of $\mathfrak{A}$ as that in which multiplication is defined by

$(27)\left(a+v^{\prime} * b\right) *\left(x+v^{\prime} * y\right)=\left\{a * x+\left(y * b S_{*}\right) * g_{1}\right\}+v^{\prime} *\left(a S_{*} * y+x * b\right)$,

$g_{1} \notin \mathfrak{F}$, cannot be equivalent to $\mathfrak{A}$, since $(27)$ is the case $U=I, V=I$, 


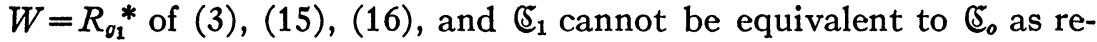
quired by Theorem 4 . For $\mathfrak{E}_{o}$ has a left unity quantity $g^{-1}$, while $\mathfrak{E}_{1}$ has a right unity quantity (the inverse of $g_{1}$ with respect to multiplication $x * y$ in $\left.\mathfrak{E}_{*}\right)$. If $\mathfrak{E}_{o} \cong \mathfrak{E}_{1}$, then $\mathfrak{E}_{o}$ has a right unity quantity $h$, and $h=g^{-1}$, $\mathfrak{S}_{o}$ has unity quantity $g^{-1}$. Then $x=x \circ g^{-1}=g x g^{-1}$, or $x g=g x$ for all $x$ in $\mathfrak{E}, g \in \mathfrak{F}$, a contradiction. Actually $\mathfrak{G}_{1}$ is antiisomorphic to $\mathfrak{E}_{0}$ in the case $\mathfrak{E}_{*} \cong{ }$ (that is, $R_{y}^{*}=P R_{y P} P^{-1}$ ) and $g_{1} P=g S$, for then $R_{y}^{(1)}=R_{y}^{*} R_{\theta_{1}}{ }^{*}=P R_{y P} R_{\sigma_{1} P} P^{-1}=P S^{2} R_{y P} R_{g S} P^{-1}$ $=P S L_{y P S} L_{0} S P^{-1}=(P S) L_{y P S}^{(o)}(P S)^{-1}$.

The automorphisms of the division algebras of order 16 over $\mathfrak{F}$ which are studied in this paper are given directly by the conditions of Theorem $4, \mathfrak{A}_{*}=\mathfrak{A}$.

THEOREM 5. A nonsingular linear transformation $H$ on a division algebra $\mathfrak{A}=\mathbb{S}+v \mathfrak{S}$ with multiplication defined by (3), yoyS not in $\mathfrak{F}$ for nonzero $y$ in $\mathfrak{E}$, is an automorphism of $\mathfrak{A}$ if and only if $H$ induces an automorphism $P$ on $\mathbb{C}$ and $\delta^{2} P$ is an automorphism of $\mathfrak{E}_{0}$ for some $\delta \neq 0$ in $\mathfrak{F}$. Such an automorphism $H$ of $\mathfrak{A}$ has the form $x+v y \leftrightarrow x P$ $+\delta v(y P)$.

Corollary. A nonsingular linear transformation $H$ on a division algebra $\mathfrak{A}=\mathfrak{S}+v \mathfrak{S}$ with multiplication defined by (4), $g \notin \mathfrak{F}$, is an automorphism of $\mathfrak{A}$ if and only if $H$ induces an automorphism $P$ on $\mathbb{S}$ such that $g P=\delta^{2} g$ for some $\delta \neq 0$ in $\mathfrak{F}$. Such an automorphism $H$ has the form $x+v y \leftrightarrow x P+\delta v(y P)$. If $t(g) \neq 0$, then $g P=g$ and either $\delta=1$ or $\delta=-1$. If $t(g)=0$, then $\delta^{4}=1$.

For $g_{1}=g$ in the corollary to Theorem 4. Moreover, $t(g P)=t(g)$, $n(g P)=n(g)$. If $t(g) \neq 0$, then $t(g P)=t\left(\delta^{2} g\right)=\delta^{2} t(g)=t(g)$ implies that $\delta^{2}=1, \delta= \pm 1$. If $t(g)=0$, then $n(g P)=n\left(\delta^{2} g\right)=\delta^{4} n(g)=n(g)$ implies that $\delta^{4}=1$.

\section{REFERENCES}

1. A. A. Albert, Quadratic forms permitting composition, Ann. of Math. (1) vol. 43 (1942) pp. 161-177.

2. R. H. Bruck, Some results in the theory of linear non-associative algebras, Trans. Amer. Math. Soc. vol. 56 (1944) pp. 141-199.

3. N. Jacobson, Cayley numbers and normal simple Lie algebras of type $G$, Duke Math. J. vol. 5 (1939) pp. 775-783.

4. R. D. Schafer, Alternative algebras over an arbitrary field, Bull. Amer. Math. Soc. vol. 49 (1943) pp. 549-555.

5. - On a construction for division algebras of order 16, Bull. Amer. Math. Soc. vol. 51 (1945) pp. 532-534.

The University of Michigan 